\title{
Post-transplant diabetes mellitus
}

\author{
Author: Tahseen A Chowdhury ${ }^{\mathrm{A}}$
}

Post-transplant diabetes mellitus (PTDM) is common following solid organ transplantation, and is a risk factor for graft failure and patient mortality. In addition to standard diabetes risk factors such as obesity and ethnicity, patients undergoing transplantation also have the additional risk factors of immunosuppressive agents and infections such as hepatitis $C$. Patients undergoing transplant assessment should be screened for diabetes. If non-diabetic, but deemed at high risk, they should be offered careful lifestyle advice to reduce risk of posttransplant weight gain and therefore reduce risk of PTDM.

Hyperglycaemia in the early post-operative period should be managed ideally with insulin therapy. Once clinically stable, there may be an opportunity to reduce or stop insulin, and consider oral hypoglycaemic agents.

Despite lack of evidence from randomised trials, PTDM should be actively screened for in all transplant recipients, and actively managed with structured education, screening for complications, cardiovascular risk reduction and antihyperglycaemic therapy.

\section{Introduction}

Since the first renal transplant between identical twins in 1954, solid organ transplantation has become an established life changing therapy for hundreds of thousands of people worldwide. With advances in immunosuppression, mortality from infection and rejection has reduced, and graft and patient survival has dramatically improved. ${ }^{1}$ Importantly, however, morbidity and mortality from cardiovascular disease is high, and a significant contributor to these adverse outcomes is post-transplant diabetes mellitus (PTDM). This was formerly known as 'new onset diabetes after transplantation' (NODAT), but the current nomenclature refers to the time of diagnosis, and recognises the fact that diabetes may well have been present pre-transplant. This distinction is important, as diabetes is a frequent pre-operative co-morbidity in many transplant patients, particularly in those undergoing renal transplantation. Depending on the type of transplant and area of study, PTDM affects anywhere between 10 and $40 \%$ of solid organ transplants. ${ }^{2}$ The diagnosis of PTDM confers a much higher risk of graft failure and patient mortality compared to those transplant patients who do not develop the

Author: ${ }^{\text {A }}$ consultant in diabetes, The Royal London Hospital, London, UK condition. ${ }^{3}$ This article aims to give an overview of the diagnosis, pathogenesis and management of PTDM.

\section{Diagnosis}

Hyperglycaemia in the post-transplant period is characterised by early post-operative hyperglycaemia, which may or may not be followed by longer-term hyperglycaemia. Early hyperglycaemia is extremely common in transplant recipients, and may be due to stress hyperglycaemia, infection, pain, immunosuppression and parenteral or enteral feeding. ${ }^{4}$ In the immediate post-transplant period where doses of immunosuppression are high, screening for PTDM should involve frequent capillary blood glucose (CBG) testing. As steroid induced hyperglycaemia is frequently a postprandial phenomenon, CBGs are best done later in the day, post lunch or evening meal.

Research in the field has been hampered by a variety of inconsistent definitions such as 'the uninterrupted need for glucose lowering therapy for at least 3 months following transplantation', 'the use of insulin postoperatively', 'the need for oral agents'

\section{Key points}

Post-transplant diabetes mellitus affects around $10-40 \%$ of patients undergoing solid organ transplantation.

The condition is associated with a higher rate of graft loss and mortality compared to patients who do not develop the condition.

Risk factors for post-transplant diabetes mellitus (PTDM) are the same as those for type 2 diabetes, but additional transplant related factors include immunosuppression (especially glucocorticoids and calcineurin inhibitors) and infection (hepatitis C and cytomegalovirus).

Early post-operative hyperglycaemia is common post-transplant and should be actively managed, ideally with insulin therapy.

Established PTDM should be treated as for type 2 diabetes, with individualised glycaemic targets, anti-hyperglycaemic therapy, cardiovascular risk reduction, screening for complications and structured diabetes care (including screening for complications).

KEYWORDS: Post-transplant diabetes, immunosuppression, transplantation = 
Table 1. Diagnostic criteria for post-transplant diabetes mellitus

$\begin{array}{llll} & \text { Diabetes } & \begin{array}{l}\text { Impaired fasting } \\ \text { glucose }\end{array} & \begin{array}{l}\text { Impaired glucose } \\ \text { tolerance }\end{array} \\ \text { Random plasma glucose } & >11.1 \mathrm{mmol} / \mathrm{L}+\text { symptoms (polyuria, } & - & - \\ \text { polydipsia, weight loss, tiredness) } & & - \\ \text { Fasting plasma glucose } & >7.0 \mathrm{mmol} / \mathrm{L} & 6.1-6.9 \mathrm{mmol} / \mathrm{L} & - \\ \text { Two-hour plasma glucose following } 75 \mathrm{~g} \text { oral glucose } & >11.1 \mathrm{mmol} / \mathrm{L} & - & 7.8-11.0 \mathrm{mmol} / \mathrm{L} \\ \text { Glycated haemoglobin (at least 3 months post-transplant) } & >6.5 \%(48 \mathrm{mmol} / \mathrm{mol}) & - & 6.0-6.4 \% \\ & & (42-47 \mathrm{mmol} / \mathrm{mol})\end{array}$

and 'fasting glucose values between $7-13 \mathrm{mmol} / \mathrm{L}$ '. International consensus guidelines have, however, established a clear method of diagnosis of the condition based on the oral glucose tolerance test (OGTT) or glycated haemoglobin (HbA1c; Table 1). ${ }^{5}$ Interpretation of $\mathrm{HbA} 1 \mathrm{c}$, however, can be problematic postoperatively, and in patients with renal disease, as $\mathrm{HbA1c}$ can be significantly affected by blood loss, iron deficiency and blood transfusion. Therefore, $\mathrm{HbA1c}$ should only be used at least 3 months post-transplantation, and prior to this, glucose tests should be undertaken.

\section{Pathogenesis}

Risk factors for the development of PTDM are the same as for type 2 diabetes (Table 2), but in addition some specific transplant related risk factors exist. These include immunosuppression and infection (such as hepatitis C virus (HCV) or cytomegalovirus $(\mathrm{CMV})$ ). Weight gain immediately post-transplant is common, and a further important risk factor for development of PTDM. Weight gain may be due to the effect of glucocorticoids, but also due to that fact that the patient feels clinically better, and may have fewer dietary restrictions compared to prior to transplantation. ${ }^{6}$

Glucocorticoids are highly diabetogenic, and will induce hyperglycaemia predominantly by exacerbating insulin resistance, enhancing gluconeogenesis and lipolysis, and reducing skeletal muscle glucose uptake. Calcineurin is an

\section{Table 2. Risk factors for development of post-}

\section{transplant diabetes mellitus}

$\begin{aligned} \text { Factors unrelated to } & >\text { Older age } \\ \text { transplantation } & >\text { South Asian / African-Caribbean ethnicity } \\ & >\text { Sedentary lifestyle } \\ & >\text { Overweight/obesity } \\ & >\text { Genetic factors } \\ & >\text { Strong family history } \\ & >\text { Adult polycystic kidney disease } \\ & >\text { Interstitial nephritis } \\ & >\text { Glucocorticoids } \\ \text { Factors associated } & >\text { Calcineurin inhibitors (tacrolimus } \\ \text { with transplantation } & >\text { ciclosporin) } \\ & >\text { Hepatitis C or cytomegalovirus infection } \\ & >\text { Post-transplant weight gain }\end{aligned}$

important factor in $\beta$-cell function and growth, and the immunosuppressant class of calcineurin inhibitors appear to have a specific adverse effect on $\beta$-cell function, leading to reduction in insulin secretion. ${ }^{7}$ While tacrolimus is an effective immunosuppressant which reduces risk of rejection, it also appears to have a more potent effect on $\beta$-cell function, leading more frequently to significant hyperglycaemia compared to other immunosuppressant regimens such as ciclosporin. A systematic review suggests that treatment of 100 renal transplant recipients with tacrolimus instead of ciclosporin avoided 12 episodes of acute rejection and two graft losses, but resulted in an extra five cases of insulin-requiring diabetes. ${ }^{8}$

Presence of CMV or HCV infection appears to increase risk of PTDM. HCV is a known risk factor for development of type 2 diabetes, and as the infection is common amongst renal and liver transplant recipients, it appears to be an important factor in PTDM. Treatment of HCV prior to transplantation may mitigate this risk. ${ }^{9}$

\section{Management}

As outlined above, early post-transplant hyperglycaemia requires active monitoring and management. Persistent hyperglycaemia (glucoses $>11 \mathrm{mmol} / \mathrm{L}$ ) should prompt treatment with intravenous or subcutaneous insulin, as there is some suggestion that early insulin therapy may reduce the risk of subsequent PTDM. ${ }^{10}$ If the patient is well, and eating and drinking, the post-prandial hyperglycaemia can be managed using neutral protamine hagedorn insulin (eg Humulin I ${ }^{\circledR}$, Insulatard ${ }^{\circledR}$, Insuman Basal ${ }^{\circledR}$ ) given in the morning, which may help to reduce glucoses later in the day. Prandial fast acting insulin may need to be added later.

As immunosuppression doses gradually reduce, hyperglycaemia frequently improves, and may resolve. Therefore, insulin doses must be reduced accordingly to avoid hypoglycaemia, and the patient must be counselled to self-test glucose levels and adjust insulin doses accordingly. Input from the diabetes specialist team, particularly diabetes specialist nursing, is mandatory.

In the absence of randomised controlled trials, the management of PTDM should follow that of type 2 diabetes. There is currently no randomised trial evidence that very tight glycaemic control will improve graft or patient outcomes in PTDM. Most guidelines suggest an $\mathrm{HbA} 1 \mathrm{c}$ target of around 7-7.5\% (53-58 mmol/ $\mathrm{mol})$, but this should be individualised according to age, comorbidity, ability to self-manage, and patient preference. ${ }^{11,12}$ It is appropriate to allow higher targets in older patients with multiple co-morbidities, as the risk of adverse effects from hypoglycaemia in such patients is very significant. 
Once the patient is on stable immunosuppression, and is clinically improving, if hyperglycaemia is persistent then there may be an opportunity to introduce oral hypoglycaemic therapy. There are few clinical trials of oral hypoglycaemic agents in PTDM, and most studies are small, single centre and non-controlled. An important consideration is the potential for drug interaction with immunosuppression, which may potentiate or reduce effects of immunosuppression. ${ }^{13}$

Of the small studies that are available, safe options for oral hypoglycaemic agents include metformin (if renal function allows), dipeptidylpeptidase-4 inhibitors (DPP-4s; of which linagliptin may be most useful as it can be used in any agree of renal function), glitazones, and meglitinides/sulfonylureas (although hypoglycaemic risk and weight gain must be considered). ${ }^{14}$ Weight gain is common post-transplant, and can sometimes be problematic. Newer agents such as glucagon-like peptide-1 (GLP-1s) are particularly useful as they can aid weight loss, and can be used in significant renal impairment. ${ }^{15}$ The potential for increased risk of genitourinary infection has led to concern over the use of sodium glucose transporter-2 (SGLT-2) inhibitors in the post-transplant setting. A recently published randomised trial of 44 patients with PTDM following renal transplantation randomised to empagliflozin or placebo showed a modest glucose benefit, but with significant weight loss, and no increase in risk of infections. ${ }^{16}$

Change in immunosuppression regimen may aid management of hyperglycaemia. If feasible, some transplant centres consider conversion of tacrolimus to ciclosporin or mycofenylate mofetil plus azathioprine in patients with difficult to control hyperglycaemia. ${ }^{17}$

In addition to standard therapy for glucose control, all patients with established PTDM must be put on to a primary care diabetes register, and undergo structured diabetes care, including referral to structured diabetes education and regular screening for complications (eyes, feet and kidneys). In addition, they require tight control of cardiovascular risk factors, such as smoking cessation, statin therapy and anti-hypertensive therapy aiming for blood pressure under 130/80 mmHg.

Patients with PTDM may be most effectively managed in a multi-disciplinary setting with diabetes and transplant specialists co-managing the patient.

\section{Prevention}

Identifying those patients at high risk of PTDM is possible using standard risk scores and specific ones for PTDM. ${ }^{18}$ Many patients wait a considerable time for transplantation, and hence this may be an opportunity to enhance knowledge about the potential for diabetes post-transplant and initiate lifestyle change. Small studies have suggested that lifestyle intervention in patients awaiting transplantation may reduce the risk of subsequent PTDM, but the answer is not definitive. ${ }^{19}$ A number of centres also encourage yearly screening for diabetes, ideally with OGTT to ensure that the patient does not develop diabetes while awaiting transplantation. While pharmacotherapy with metformin, orlistat or acarbose are all effective means of preventing diabetes in patients with impaired glucose tolerance, none of these agents have as yet been studied in the transplant setting. Treatment of HCV infection prior to transplantation may reduce risk of PTDM. ${ }^{9}$ As stated before, one study of early insulin therapy treating early hyperglycaemia immediately after transplantation has been found to reduce risk of PTDM by $73 \% .{ }^{10}$ Confirmatory studies are awaited.

\section{Conclusions}

PTDM is common following solid organ transplantation, and is an important risk marker for early and late graft failure, and early and late mortality. Immediate post-transplant hyperglycaemia requires active monitoring and management with insulin therapy, and if persistent once the patient is medical stable, there may be an opportunity to reduce or stop insulin, and introduce oral hypoglycaemic therapy. Once PTDM is established, treatment targets and pathways should be as for type 2 diabetes. There is an opportunity to consider prevention of PTDM in high-risk individuals while the person is awaiting transplantation.

\section{References}

1 Saran R, Li Y, Robinson B et al. US Renal Data System 2014 annual data report: epidemiology of kidney disease in the United States. Am J Kidney Dis 2015;66(Suppl 1):S1-306.

2 Yates C], Fourlanos S, Hejlmesaeth J, Colman PG, Cohney SJ. New onset diabetes after kidney transplantation - changes and challenges. Am J Transplant 2012;12:820-8.

3 Kuo HT, Sampaio MS, Vincenti F, Bunnapradist S. Associations of pretransplant diabetes mellitus, new-onset diabetes after transplant, and acute rejection with transplant outcomes: an analysis of the Organ Procurement and Transplant Network/United Network for Organ Sharing (OPTN/UNOS) database. Am J Kidney Dis 2010;56:1127-39.

4 Gupta S, Pollack T, Fulkerson C et al. Hyperglycemia in the Posttransplant Period: NODAT vs Posttransplant Diabetes Mellitus. J Endocr Soc 2018:2:1314-9.

5 Sharif A, Heckin M, de Vries APR et al. Proceedings from an international consensus meeting on posttransplant diabetes mellitus: recommendations and future directions. Am J Transplant 2014;:14:1992-2000.

6 Rodrigo E, Santos L, Piñera C et al. Prediction at first year of incident new-onset diabetes after kidney transplantation by risk prediction models. Diabetes Care 2012;35:471-3.

7 Øzbay LA, Smidt K, Mortensen DM et al. Cyclosporin and tacrolimus impair insulin secretion and transcriptional regulation in INS-1E beta-cells. Br J Pharmacol 2011;162:136-46.

8 Webster AC, Woodroffe RC, Tayloer RS, Chapman JR, Craig JC. Tacrolimus versus cyclosporin as primary immunosuppression for kidney transplant recipients: meta-analysis and meta-regression of randomised trial data. BMJ 2005;331:810.

9 Roccaro GA, Mitrani R, Hwang WT, Forde KA, Reddy KR. Sustained virological response is associated with a decreased risk of posttransplant diabetes mellitus in liver transplant recipients with hepatitis C-related liver disease. Liver Transp/ 2018;24:1665-72.

10 Hecking M, Haidinger M, Döller D et al. Early basal insulin therapy decreases new-onset diabetes after renal transplantation. J Am Soc Nephrol 2012;23:739-49.

11 American Diabetes Association. Glycaemic targets: standards of medical care in diabetes. Diabetes Care 2018:41(Suppl 1):S55-64.

12 Kasiske B, Zeier MG, Chapman JR et al. KDIGO clinical practice guideline for the care of kidney transplant recipients: a summary. Kidney Int 2010;77:299-311.

13 Vanhove T, Remijsen Q, Kuypers D, Gillard P. Drug-drug interactions between immunosuppressants and antidiabetic drugs in the treatment of post-transplant diabetes mellitus. Transplant Rev (Orlando) 2017;31:69-77.

14 Jenssen T, Hartmann A. Post-transplant diabetes mellitus in patients with solid organ transplants. Nat Rev Endocrinol 2019;15:172-88.

15 Singh P, Pesavento TE, Washburn K, Walsh D, Meng S. Largest single-centre experience of dulaglutide for management of diabetes mellitus in solid organ transplant recipients. Diabetes Obes Metab 2019:21:1061-5. 
16 Halden TAS, Kvitne KE, Midtvedt K et al. Efficacy and safety of empagliflozin in renal transplant recipients with posttransplant diabetes mellitus. Diabetes Care 2019;42:1067-74.

17 Oberholzer ], Thielke J, Hatipoglu B et al. Immediate conversion from tacrolimus to cyclosporine in the treatment of posttransplantation diabetes mellitus. Transplant Proc 2005:37:999_ 1000.

18 Chakkera HA, Weil EJ, Swanson CM et al. Pretransplant risk score for new-onset diabetes after kidney transplantation. Diabetes Care 2011;34:2141-5
19 Sharif A, Moore R, Baboolal K. Influence of lifestyle modification in renal transplant recipients with postprandial hyperglycemia. Transplantation 2008;85:353-8.

Address for correspondence: Prof Tahseen A Chowdhury, Department of Diabetes and Metabolism, 7th Floor, John Harrison House, The Royal London Hospital, Whitechapel, London E1 1BB, UK.

Email: tahseen.chowdhury@nhs.net

\section{Royal College} of Physicians

\section{Medical Care} Are you involved in planning
diabetes and endocrinology services?

Medical Care is a comprehensive web-based resource for the efficient and effective design of medical services covering all the specialties, including diabetes and endocrinology.

The resource looks in detail at the services delivered by diabetes and endocrinology, including:

> inpatient and outpatient care

$>$ pregnancy and diabetes

$>$ integrated diabetes services

$>$ diabetes renal services.
Medical Care has been designed to help those involved in the planning and provision of medical services get a clearer picture of the specialty services that need to be in place to provide great patient care.

\section{www.rcpmedicalcare.org.uk}

